\title{
Влияние шероховатости поверхности на смену режима роста с двумерного на трехмерный в напряженных SiGe-гетероструктурах
}

\author{
(C) А.В. Новиков ${ }^{+*}$, М.В. Шалеев ${ }^{+}$, Д.В. Юрасов ${ }^{+*}$, П.А. Юнин ${ }^{+*}$ \\ + Институт ффизики микроструктур Российской академии наук, \\ 603950 Нижний Новгород, Россия \\ * Нижегородский государственный университет им. Н.И. Лобачевского, \\ 603950 Нижний Новгород, Россия \\ E-mail: anov@ipmras.ru
}

(Получена 27 апреля 2016 г. Принята к печати 10 мая 2016 г.)

Исследовано влияние микрошероховатости поверхности роста на критическую толщину двумерного роста напряженных $\mathrm{SiGe}$-структур, выращенных на подложках $\mathrm{Si}(001)$ и $\mathrm{Ge}(001)$. Для решеток $\mathrm{Ge} / \mathrm{Si}$, выращенных на подложках $\mathrm{Si}(001)$, обнаружено уменьшение критической толщины двумерного роста Ge при увеличении числа периодов решетки или уменьшении толщины разделительных слоев $\mathrm{Si}$, которое связывается с увеличением шероховатости поверхности роста по мере накопления в сжатых структурах упругой энергии. Сравнительные исследования роста $\mathrm{SiGe-cтруктур} \mathrm{на} \mathrm{подложках} \mathrm{Si}(001)$ и $\mathrm{Ge}(001)$ показали, что в широком диапазоне составов слоев $\mathrm{SiGe}$ при одинаковом по абсолютной величине рассогласовании кристаллических решеток пленки и подложки критическая толщина двумерного роста растянутых слоев значительно больше, чем сжатых.

\section{1. Введение}

Хорошо известно, что для широкого диапазона составов напряженных (сжатых) структур $\mathrm{Si}_{1-x} \mathrm{Ge}_{x} / \mathrm{Si}(001)$ упругая релаксация напряжений через образование самоформирующихся наноостровков предшествует пластической релаксации за счет формирования дефектов (см., например, обзоры $[1,2])$. Однако сам механизм зарождения наноостровков несколько различен для структур с большой и малой долей $\mathrm{Ge}$. Для слоев $\mathrm{Si}_{1-x} \mathrm{Ge}_{x}$ с небольшой долей $\mathrm{Ge}(x<80 \%)$ формирование островков происходит по так называемому „безбарьерному“ механизму, за счет постепенного развития шероховатости поверхности и образования на начальных этапах трехмерного роста неограненных островков (mounds) [3,4]. По мере роста толщины осажденной пленки $\mathrm{SiGe}$ неограненные островки трансформируются в пирамидальные с боковыми гранями типа $(105)[3,4]$. $\mathrm{B}$ то же время для слоев $\mathrm{Si}_{1-x} \mathrm{Ge}_{x}$ с большой долей $\mathrm{Ge}$ $(x>80 \%)$, для которых критическая толщина двумерного роста составляет единицы монослоев (1 монослой (MC) соответствует $\sim 0.14 \mathrm{HM}$ ), такое постепенное развитие шероховатости поверхности роста и формирование неограненных островков в однослойных структуpax было обнаружено лишь в единичных работах при очень низких скоростях осаждения (<0.05 МС/мин) [5]. В большинстве же работ наблюдалось сохранение малой шероховатости поверхности таких слоев вплоть до момента образования пирамидальных островков. Это означает, что для формирования островков в слоях $\mathrm{Si}_{1-x} \mathrm{Ge}_{x} / \mathrm{Si}(001)$ с большой долей $\mathrm{Ge}$ необходимо преодоление некоторого энергетического барьера [6]. Наличие такого барьера затрудняет зарождение островков. В результате экспериментально определяемая критическая толщина двумерного роста $\left(h_{c}\right)$ Ge на $\mathrm{Si}(001)$ (4-5MC) оказывается больше равновесной толщины смачивающего слоя (3 МС) [7]. Очевидно, что увеличение (уменьшение) микрошероховатости поверхности роста облегчит (затруднит) преодоление энергетического барьера и приведет к уменьшению (увеличению) критической толщины двумерного роста напряженных слоев $\mathrm{Si}_{1-x} \mathrm{Ge}_{x}$. При росте $\mathrm{SiGe-гетероструктур} \mathrm{на} \mathrm{подложках}$ с кристаллографической ориентацией (001) управление микрошероховатостью поверхности роста возможно за счет осаждения слоев с различным знаком деформации. Это вызвано различным влиянием сжатых и растянутых слоев на энергию образования моноатомных ступеней на реконструированной $(2 \times 1)$ поверхности (001) [8]: деформация сжатия понижает энергию, а деформация растяжения повышает. В результате предосаждение сжатых планарных слоев $\mathrm{SiGe}$ приводит к росту микрошероховатости поверхности и, как следствие, к уменьшению величины $h_{c}$ при росте $\mathrm{Ge}$ на $\mathrm{Si}(001)$ [9], а растянутых слоев $\mathrm{Si}$ - к „выглаживанию“ поверхности роста и увеличению $h_{c}$ при росте Ge на релаксированных буферах $\mathrm{SiGe} / \mathrm{Si}(001)$ [10]. При этом влияние предосажденных напряженных слоев $\mathrm{SiGe}$ на дальнейший рост гетероструктуры сохраняется и при их заращивании тонким ненапряженным слоем матрицы [9,10]. Это указывает на то, что в многослойных SiGe-структурах, в которых напряженные планарные слои разделены между собой тонкими слоями ненапряженного материала, характер релаксации упругих напряжений может меняться по мере роста структуры. В настоящей работе данное предположение экспериментально проверено на примере напряженных решеток $\mathrm{Ge} / \mathrm{Si}$, выращенных на подложках $\mathrm{Si}(001)$. Выявлена смена механизма зарождения самоформирующихся наноостровков Ge c „барьерного“ на „безбарьерный“ по мере роста числа периодов решетки и/или уменьшения толщины разделительных слоев $\mathrm{Si}$. 
Зависимость для гетеросистемы $\mathrm{Ge} / \mathrm{Si}$ шероховатости поверхности (001) от знака деформации [8] должна приводить к существенному различию в релаксации упругих напряжений сжатых и растянутых слоев $\mathrm{Si}_{1-x} \mathrm{Ge}_{x}$, что и было экспериментально показано в ряде работ $[8,11,12]$. Однако процессы релаксации упругих напряжений за счет развития шероховатости для растянутых слоев $\mathrm{GeSi}$, выращенных на подложках $\mathrm{Ge}(001)$, к настоящему времени остаются исследованными в гораздо меньшей степени, чем для SiGe-структур, выращенных на подложках $\mathrm{Si}(001)$. В данной работе исследован рост напряженных слоев $\mathrm{GeSi} / \mathrm{Ge}(001)$ и показано, что в широком диапазоне составов слоев SiGe при одинаковом по величине рассогласовании кристаллических решеток пленки и подложки критическая толщина двумерного роста растянутых слоев $\mathrm{GeSi} / \mathrm{Ge}(001)$ значительно больше, чем сжатых $\mathrm{SiGe} / \mathrm{Si}(001)$.

\section{2. Методика эксперимента}

Исследованные структуры были выращены на подложках $\mathrm{Si}(001)$ и $\mathrm{Ge}(001)$ методом молекулярно-пучковой эпитаксии (МПЭ). Температура роста SiGe-структур на подложках $\mathrm{Si}(001)$ составляла $700^{\circ} \mathrm{C}$, а на $\mathrm{Ge}(001)-$ $600^{\circ} \mathrm{C}$. Выбор температур роста был обусловлен минимизацией влияния на рост структур двух факторов: кинетических ограничений, проявляющихся при низких температурах, и диффузионного перемешивания слоев, ярко выраженного при высоких температурах. Более низкая температура роста структур на подложках $\mathrm{Ge}(001)$ связана с более высокой поверхностной диффузией атомов $\mathrm{Si}$ и $\mathrm{Ge}$ на $\mathrm{Ge}(001)$ по сравнению с диффузией на $\mathrm{Si}(001)$ [13]. Скорость осаждения $\mathrm{Ge}$ при росте на

\begin{tabular}{|c|}
\hline Ge layer, $d_{\mathrm{Ge}}$ \\
\hline Si spacer, $d_{\mathrm{Si}}$ \\
\hline Ge layer, $d_{\mathrm{Ge}}$ \\
\hline Si spacer, $d_{\mathrm{Si}}$ \\
\hline Ge layer, $d_{\mathrm{Ge}}$ \\
\hline Si buffer \\
\hline $\operatorname{Si}(001)$ \\
\end{tabular}

Рис. 1. Схематичное изображение решеток $\mathrm{Ge} / \mathrm{Si}$, исследованных в работе. $d_{\mathrm{Ge}}-$ толщина слоя $\mathrm{Ge}, d_{\mathrm{Si}}-$ толщина разделительного слоя $\mathrm{Si}, N$ - число периодов решетки. подложках $\mathrm{Si}(001)$ составляла $\sim 0.02 \mathrm{HM} / \mathrm{c}$, а слоев $\mathrm{SiGe}$ на подложках $\mathrm{Ge}(001) \sim 0.1 \mathrm{Hм} / \mathrm{c}$. В случае роста на подложках $\mathrm{Si}(001)$ исследованные структуры состояли из буферного слоя $\mathrm{Si}$ толщиной $100 \mathrm{Hм} \mathrm{и} \mathrm{решетки}$ $\mathrm{Ge} / \mathrm{Si}$. Схематично изображение исследованных решеток представлено на рис. 1. Толщина слоев $\mathrm{Ge}$ в различных решетках варьировалась в пределах $d_{\mathrm{Ge}}=2-4 \mathrm{MC}$, а разделительных слоев $\mathrm{Si}-d_{\mathrm{Si}}=2-6$ нм. Рост решеток $\mathrm{Ge} / \mathrm{Si}$ заканчивался при переходе слоя $\mathrm{Ge}$ к островковому росту. При росте на подложках $\mathrm{Ge}(001)$ структуры состояли из буферного слоя $\mathrm{Ge}$ и слоя $\mathrm{Ge}_{x} \mathrm{Si}_{1-x}$ с долей $\mathrm{Ge} x=20-65 \%$ толщиной $d_{\mathrm{GeSi}}=1-13$ нм. Рост структур (перестройка поверхности и критическая толщина двумерного роста $h_{c}$ ) контролировался in situ с помощью дифракции быстрых электронов (ДБЭ). Критическая толщина двумерного роста напряженных слоев определялась также ex situ с помощью атомно-силовой микроскопии (ACM) на микроскопе NTEGRA PRIMA (НТ-МДТ). АСМ использовалась также для исследования морфологии поверхности выращенных структур. Состав и степень релаксации упругих напряжений в структурах $\mathrm{GeSi} / \mathrm{Ge}(001)$ определялись с помощью рентгеновского дифракционного анализа.

\section{3. Результаты и их обсуждение}

\section{1. Рост решеток $\mathrm{Ge} / \mathrm{Si}$ на подложках $\mathrm{Si}(001)$}

Для решеток $\mathrm{Ge} / \mathrm{Si}$, состоящих из слоев $\mathrm{Ge}$ толщиной $d_{\mathrm{Ge}}=2-4 \mathrm{MC}$, разделенных слоями $\mathrm{Si}$ толщиной $d_{\mathrm{Si}}=2-6$ нм, обнаружено уменьшение критической толщины двумерного роста Ge при увеличении числа периодов решетки $(N)$ и/или уменьшении толщины разделительных слоев $\mathrm{Si}\left(d_{\mathrm{Si}}\right)$. Так, если в однослойных структурах $\mathrm{Ge} / \mathrm{Si}(001)$ согласно ДБЭ $h_{c}=4.5-5 \mathrm{MC}$, то для решетки $\mathrm{Ge} / \mathrm{Si} 4 \mathrm{MC} / 6 \mathrm{Hм}$ образование островков наблюдается уже во втором периоде, а для $3 \mathrm{MC} / 2 \mathrm{Hм}-$ в третьем, т.е. значения $h_{c}$ для второго и третьего периодов этих решеток составляют 4 и 3 МС соответственно. При этом увеличение толщины разделительного слоя $\mathrm{Si}$ до $d_{\mathrm{Si}}=3$ нм в решетке $\mathrm{Ge} / \mathrm{Si}$ со слоями $\mathrm{Ge}$ толщиной $d_{\mathrm{Ge}}=3 \mathrm{MC}$ приводит к отсутствию островков в третьем периоде решетки $\left(h_{c}>3 \mathrm{MC}\right)$. Выявленное уменьшение $h_{c}$ пленки $\mathrm{Ge}$ связывается с развитием микрошероховатости поверхности при росте решеток $\mathrm{Ge} / \mathrm{Si}$ с увеличением накопленной в них упругой энергии. Данное предположение подтверждается результатами АСМ-исследований, показавшими, что слои $\mathrm{Ge}$ одинаковой толщины $\left(<h_{c}\right)$ в первом и верхних слоях решеток $\mathrm{Ge} / \mathrm{Si}$ имеют различную морфологию поверхности (рис. 2). Так, на АCM-снимке слоя Ge толщиной $d_{\mathrm{Ge}}=2.5 \mathrm{MC}$, осажденного на буфер $\mathrm{Si}$ („первого“ слоя в решетке $\mathrm{Ge} / \mathrm{Si} 2.5 \mathrm{MC} / 2 \mathrm{HM})$, не наблюдается никаких особенностей (рис. 2,a). При этом шероховатость поверхности слоя Ge близка к шероховатости поверхности буфера Si (среднеквадратичная шероховатость поверхности, определенная из АСМ-снимка, $\sim 0.2 \mathrm{Hм})$. В то 

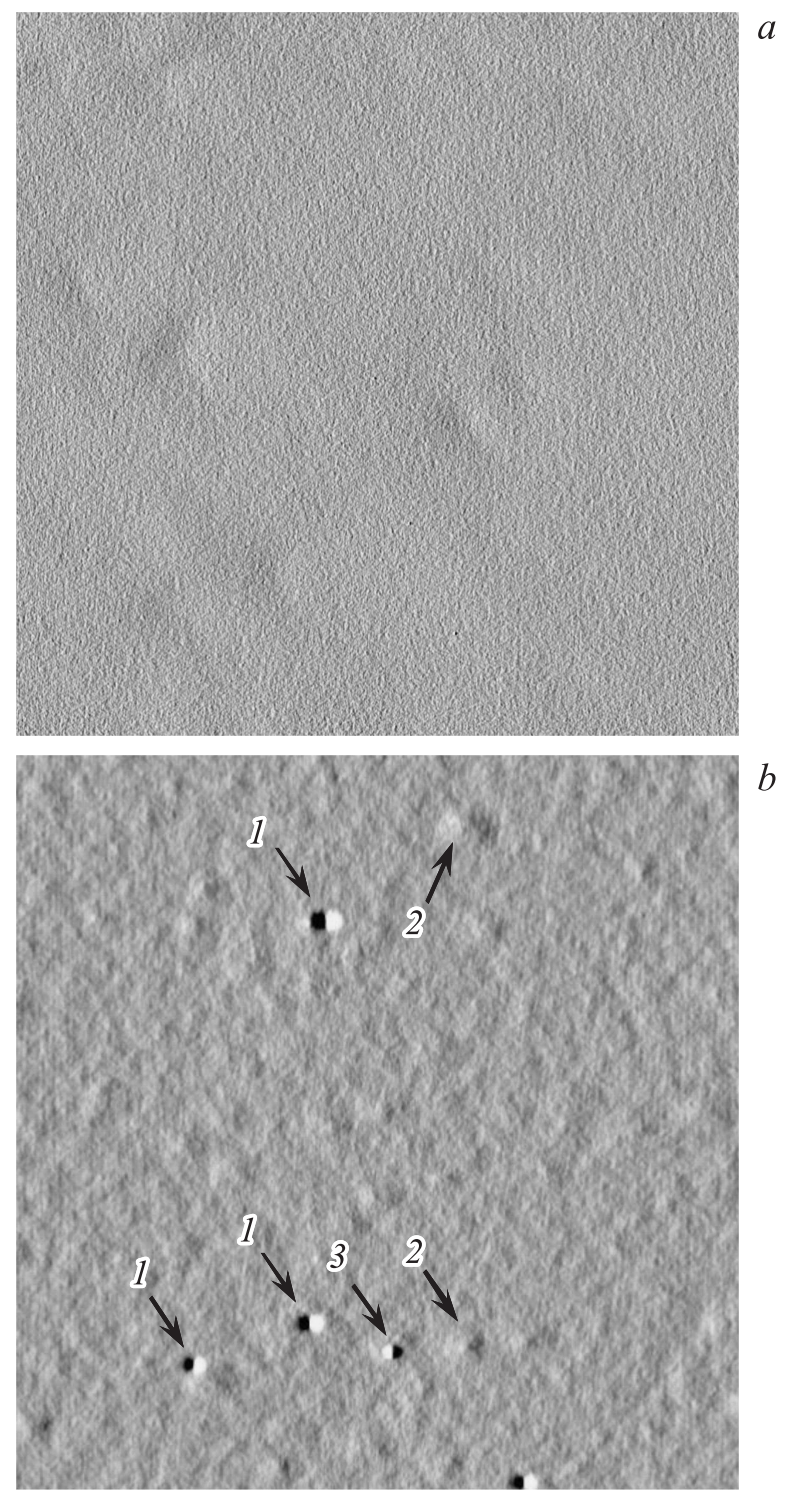

Рис. 2. АСМ-снимки поверхности однослойной структуры $\mathrm{Ge} / \mathrm{Si}(001)$ со слоем $\mathrm{Ge}$ толщиной $2.5 \mathrm{MC}(a)$ и шестого слоя $\mathrm{Ge}$ в решетке $\mathrm{Ge} / \mathrm{Si} 2.5 \mathrm{MC} / 2$ нм $(b)$. Снимки обработаны фильтром Прюитта для подчеркивания микрошероховатости поверхности. $b$ : отмечены ямки (pits) $(1)$, неограненные возвышенности (mounds) (2) и мелкие островки (pre-pyramids) (3). Размеры снимков $3 \times 3$ мкм.

же время на поверхности слоя Ge такой же толщины в шестом периоде решетки при незначительном увеличении микрошероховатости (среднеквадратичная шероховатость поверхности выросла до $\sim 0.3$ нм) наблюдается формирование мелких ямок (pits), неограненных возвышенностей (mounds) и мелких островков (pre-pyramids) (рис. 2,b). Данный вид поверхности типичен для напряженных слоев $\mathrm{Si}_{1-x} \mathrm{Ge}_{x} / \mathrm{Si}(001)(x<80 \%)$, толщина которых близка к критической толщине их двумерного роста [4]. Полагается, что развитие шероховатости поверхности (в частности, формирование неограненных островков) по мере роста числа периодов решетки $\mathrm{Ge} / \mathrm{Si}$ меняет механизм зарождения островков с „барьерного“, характерного для первых периодов решетки, на „безбарьерный“ в ее верхних слоях. Отсутствие энергетического барьера на формирование островков в последнем случае ведет к наблюдаемому уменьшению критической толщины двумерного роста германия в решетке $\mathrm{Ge} / \mathrm{Si}$. Как видно из рис. 2, $b$, образование неограненных возвышенностей (mounds) и мелких островков (pre-pyramids) в исследуемых решетках $\mathrm{Ge} / \mathrm{Si}$ наблюдалось и при толщине $\mathrm{Ge}<3 \mathrm{MC}$, которая для однослойных структур считается термодинамически равновесной толщиной смачивающего слоя $\mathrm{Ge}$ на $\mathrm{Si}(001)$ (см., например, [7]). Данное наблюдение согласуется с ранее полученными результатами, свидетельствующими о возможности формировании островков в многослойных SiGe-структурах, толщина отдельных слоев Ge в которых < 3 MC [9].

Необходимо отметить, что количественно зависимость $h_{c}$ от параметров решеток $\mathrm{Ge} / \mathrm{Si}$ достаточно хорошо описывается моделью [14], развитой для описания влияния предосаждения напряженных $\mathrm{SiGe} / \mathrm{Si}(001)$ слоев на последующий рост пленки Ge [9]. В данной модели была предложена новая оценка величины $h_{c}$, основанная на накоплении в напряженных структурах эффективной упругой энергии до некой критической величины [14]. В расчетах учитывается упругая энергия, накопленная в напряженных подслоях $\mathrm{SiGe}$, находящихся на расстоянии 20-30 MC (3-4 нм) от поверхности. Согласно экспериментальным результатам [9], именно таков масштаб толщин, на котором заращённые напряженные подслои оказывают влияние на дальнейший рост SiGe-структур. В модели также учитывается изменение состава осаждаемых слоев $\mathrm{SiGe}$ в результате сегрегации $\mathrm{Ge}$ [14]. Согласно расчетам, выполненным с использованием модели из работы [14], при экспериментальном значении $h_{c}=4.5 \mathrm{MC}$ для роста $\mathrm{Ge}$ на $\mathrm{Si}(001)$ накопленная упругая энергия в структуре, необходимая для формирования островков, составляет $\sim 0.1$ Дж/ $\mathrm{M}^{2}$. Анализ зависимости $h_{c}$ от параметров решеток $\mathrm{Si} / \mathrm{Ge}$, исследованных в данной работе, показывает, что образование островков в верхних слоях решеток начинается при накоплении в них эффективной упругой энергии

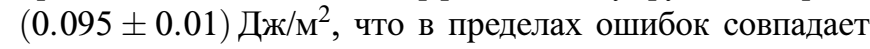
с критической упругой энергией для роста $\mathrm{Ge}$ на $\mathrm{Si}(001)$. Исходя из этого можно предположить, что выявленное ранее уменьшение $h_{c}$ при росте Ge в структурах с напряженными подслоями $\mathrm{SiGe}$ также связано с влиянием микрошероховатости слоя $\mathrm{SiGe}$ на механизм зарождения островков Ge. В этом случае введенный в работах $[9,14]$ феноменологический параметр $L_{\mathrm{dec}}$, характеризующий масштаб толщин, на котором предосажденный слой $\mathrm{SiGe}$ оказывает влияние на последующий рост структур, может быть связан с масштабом толщин, на котором при используемых условиях роста происходит или развитие (при осаждении напряженного подслоя $\mathrm{SiGe}$ ), или уменьшение (при его заращивании ненапряженным слоем $\mathrm{Si}$ ) микрошероховатости поверхности роста. 

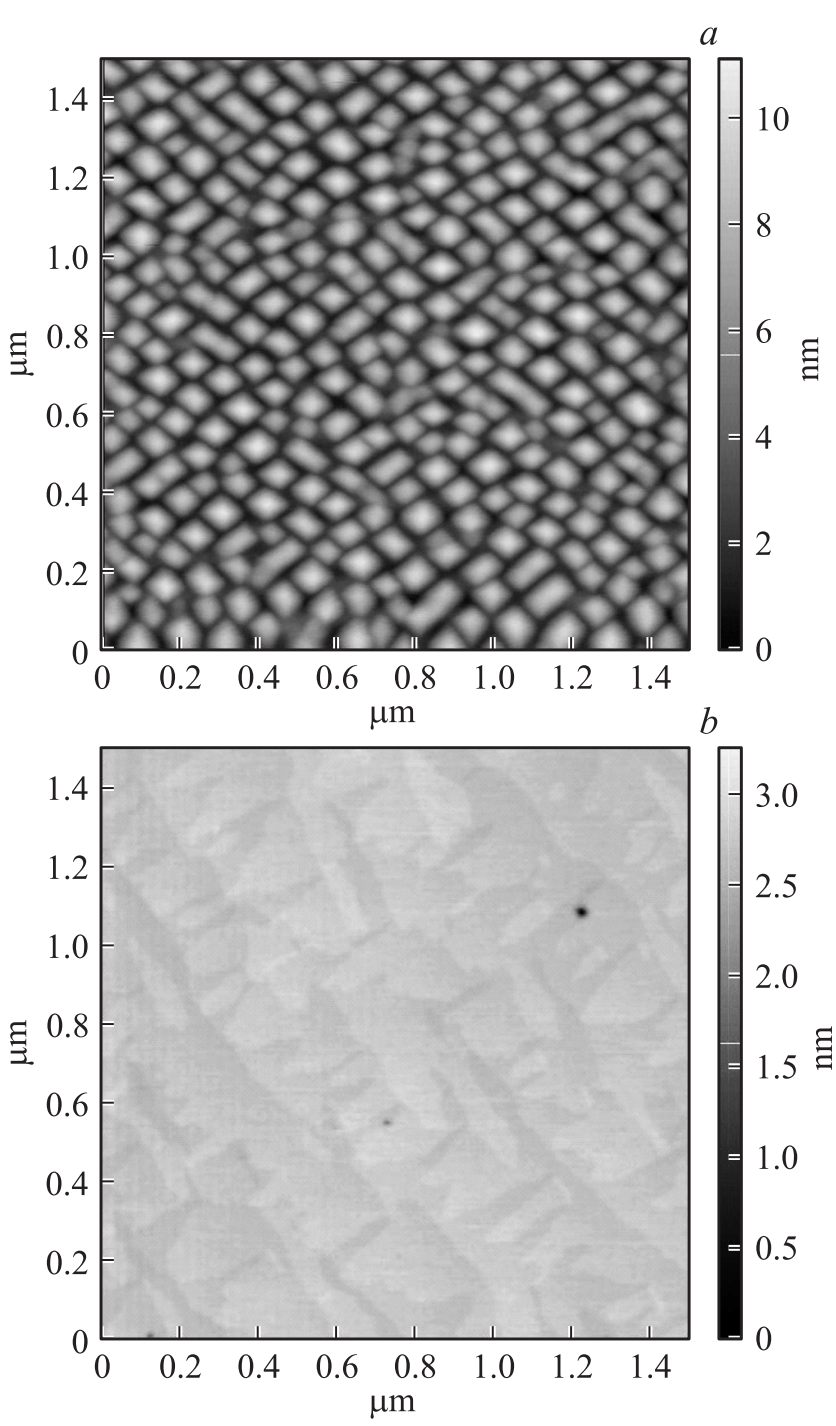

Рис. 3. АСМ-снимки слоя $\mathrm{Si}_{0.65} \mathrm{Ge}_{0.35} / \mathrm{Si}(001)$ толщиной 3.5 нм $(a)$ и слоя $\mathrm{Si}_{0.35} \mathrm{Ge}_{0.65} / \mathrm{Ge}(001)$ толщиной 8 нм $(b)$. Боковые стороны снимка ориентированы: $a$ - вдоль направлений [110], $b$ - вдоль направления типа [010].

\section{2. Релаксация упругих напряжений в слоях $\mathrm{GeSi}$, выращенных на подложках $\mathrm{Ge}(001)$}

Для исследования особенностей релаксации упругих напряжений в растянутых слоях за счет смены механизма роста с двумерного на трехмерный на подложках $\mathrm{Ge}(001)$ была выращена серия структур со слоями $\mathrm{Si}_{1-x} \mathrm{Ge}_{x}$. Доля $\mathrm{Ge}$ в слоях варьировалась от 20 до $65 \%$, а их толщина - от 1 до 13 нм. Результаты АСМи ДБЭ-исследований структур на подложках $\mathrm{Ge}(001)$ сравнивались с результатами исследований структур с сжатыми слоями $\mathrm{SiGe}$, выращенными на подложках $\mathrm{Si}(001)$ и имеющими близкую по абсолютной величине упругую деформацию. Установлено, что в широком диапазоне составов критическая толщина двумерного роста растянутых слоев $\mathrm{GeSi} / \mathrm{Ge}(001)$ больше, чем сжатых $\mathrm{SiGe} / \mathrm{Si}(001)$. Так, для слоя $\mathrm{Si}_{1-x} \mathrm{Ge}_{x} / \mathrm{Si}(001)$ с долей $\mathrm{Ge} x=35 \%$, что соответствует абсолютному значению деформации сжатия $\varepsilon \approx 1.5 \%$, критическая толщина двумерного роста составляет $h_{c}=3.5 \mathrm{Hм}$, т.е. $\sim 25$ МС. При этой толщине слоя $\mathrm{Si}_{1-x} \mathrm{Ge}_{x}$ на поверхности наблюдается плотный массив пирамидальных островков (рис. 3,a). В то же время поверхность слоя $\mathrm{Si}_{1-x} \mathrm{Ge}_{x} / \mathrm{Ge}(001)$ с долей $\mathrm{Ge} x=65 \%$, что соответствует абсолютному значению деформации растяжения $\varepsilon \approx 1.5 \%$, остается планарной и при толщине слоя в 8 нм (рис. $3, b$ ). При этом на АСМ-снимке этого слоя хорошо различимы моноатомные ступени с широкими террасами между ними (рис. $3, b$ ), а шероховатость поверхности остается $<0.2$ нм. Следовательно, при росте растянутых слоев SiGe, в отличие от сжатых, не происходит развития микрошероховатости поверхности роста. Данный факт связывают с увеличением энергии образования моноатомных ступеней на реконструированной $(2 \times 1)$ поверхности $(001)$ в системе $\mathrm{Si} / \mathrm{Ge}$ c ростом деформации растяжения и уменьшением энергии с ростом деформации сжатия [8]. Малая шероховатость поверхности растянутых слоев понижает вероятность зарождения самоформирующихся трехмерных объектов и, как следствие, увеличивает критическую толщину двумерного роста структур $\mathrm{GeSi} / \mathrm{Ge}(001)$. Это особенно ярко выражено для напряженных слоев с небольшой деформацией $(\varepsilon<2.5 \%)$, для которых в случае сжатых слоев $\mathrm{SiGe} / \mathrm{Si}(001)$ образование островков идет через постепенное развитие шероховатости поверхности $[3,4]$.

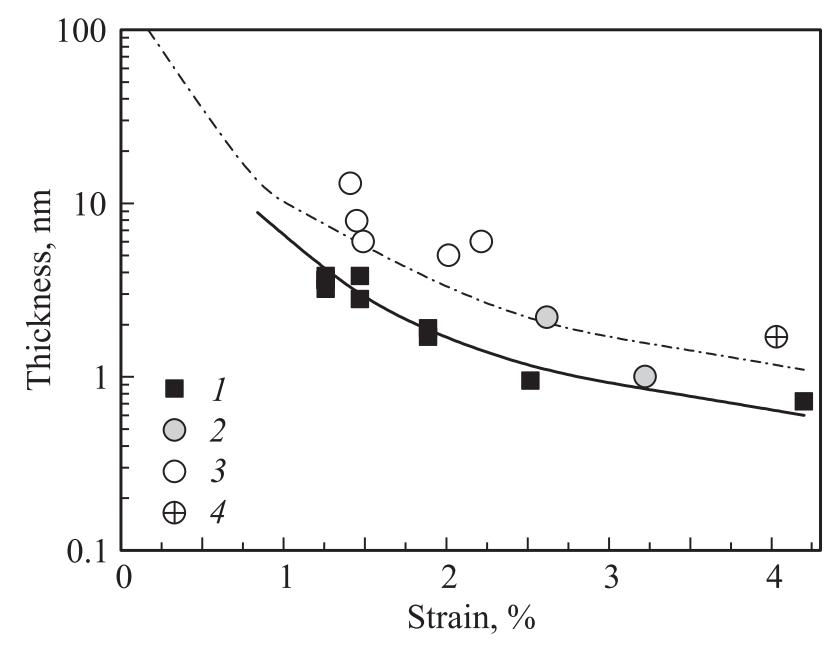

Рис. 4. Зависимости критической толщины двумерного и псевдоморфного роста слоев $\mathrm{SiGe} / \mathrm{Si}(001)$ и $\mathrm{GeSi} / \mathrm{Ge}(001)$ от абсолютного значения деформации. 1 - экспериментальные данные по $h_{c}$ для сжатых слоев $\mathrm{SiGe} / \mathrm{Si}(001)$; сплошная кривая - экспериментальная зависимость $h_{c}$ для сжатых $\mathrm{SiGe} / \mathrm{Si}(001)$ слоев из работы $[1] ; 2-h_{c}$ для растянутых слоев $\mathrm{GeSi} / \mathrm{Ge}(001) ; 3$ - параметры растянутых слоев $\mathrm{GeSi} / \mathrm{Ge}(001)$, в которых не наблюдалось перехода к трехмерному росту; 4 - экспериментальная точка для критической толщины двумерного роста $\mathrm{Si}$ на $\mathrm{Ge}(001)$ из работы [12]. Штрихпунктирная кривая - теоретически рассчитанная зависимость критической толщины псевдоморфного роста из работы [15]. 
В результате существует широкий диапазон составов слоев, в котором критическая толщина двумерного роста растянутых слоев $\mathrm{GeSi} / \mathrm{Ge}(001)$ не только больше, чем сжатых $\mathrm{SiGe} / \mathrm{Si}(001)$, но и превосходит критическую толщину их псевдоморфного роста, рассчитанную в работе [15] (рис. 4). При толщине слоя $\mathrm{SiGe,} \mathrm{большей}$ критической толщины псевдоморфного роста, становится энергетически выгодным образование дефектов (дислокаций) в растущей пленке. Из-за того что величина $h_{c}$ в структурах $\mathrm{GeSi} / \mathrm{Ge}(001)$ больше критической толщины начала пластической релаксации упругих напряжений, трехмерные самоформирующиеся объекты, образующиеся в этих структурах, содержат дефекты кристаллической решетки (дислокации) $[11,12]$.

\section{4. Заключение}

B работе для SiGe-структур, выращенных методом МПЭ на подложках $\mathrm{Si}(001)$ и $\mathrm{Ge}(001)$, исследовано влияние микрошероховатости поверхности на смену механизма роста напряженных слоев с двумерного на трехмерный. Для решеток $\mathrm{Ge} / \mathrm{Si}$, выращенных на подложках $\mathrm{Si}(001)$ и состоящих из слоев $\mathrm{Ge}$ толщиной 2-4 MC, разделенных слоями $\mathrm{Si}$ толщиной 2-6нм, обнаружено уменьшение критической толщины двумерного роста Ge при увеличении числа периодов решетки и/или уменьшении толщины разделительных слоев $\mathrm{Si}$. Уменьшение критической толщины двумерного роста Ge при увеличении числа периодов решетки или уменьшении толщины разделительных слоев $\mathrm{Si}$ связывается с развитием шероховатости поверхности роста по мере накопления в структуре упругой энергии, что понижает энергетический барьер на образование самоформирующихся островков. Для SiGe-структур, выращенных на подложках $\mathrm{Ge}(001)$, показано, что в широком диапазоне составов слоев $\mathrm{SiGe}$ при одинаковом по абсолютной величине рассогласовании кристаллических решеток пленки и подложки критическая толщина двумерного роста растянутых слоев $\mathrm{GeSi} / \mathrm{Ge}(001)$ значительно больше, чем сжатых $\mathrm{SiGe} / \mathrm{Si}(001)$. Рост критической толщины двумерного роста для растянутых слоев $\mathrm{SiGe}$ связывается с увеличением энергии образования моноатомных ступеней на реконструированной $(2 \times 1)$ поверхности (001) с ростом деформации растяжения.

Работа выполнена при поддержке научных программ РАН и грантов РФФИ № 16-52-50017-ЯФ_а и 14-02-01116.

\section{Список литературы}

[1] K. Brunner. Rep. Progr. Phys., 65, 27 (2002).

[2] J.-N. Aqua, I. Berbezier, L. Favre, T. Frisch, A. Ronda. Phys. Reports, 522, 59 (2013)

[3] J. Tersoff, B.J. Spencer, A. Rastelli, H. von Kanel. Phys. Rev. Lett., 89, 196104 (2002).

[4] A. Rastelli, H. von Kanel. Surf. Sci., 532, 769 (2003).
[5] A. Vailionis, B. Cho, G. Glass, P. Desjardins, D.G. Cahill, J.E. Greene. Phys. Rev. Lett., 85, 3672 (2000).

[6] F.M. Ross, J. Tersoff, R.M. Tromp. Phys. Rev. Lett., 80, 984 (1998).

[7] M. Brehm, F. Montalenti, M. Grydlik, G. Vastola, H. Lichtenberger, N. Hrauda, M.J. Beck, Th. Fromherz, F. Schäffler, L. Miglio, G. Bauer. Phys. Rev. B, 80, 205321 (2009).

[8] Y.H. Xie, G.H. Gilmer, C. Roland, P.J. Silverman, S.K. Buratto, J.Y. Cheng, E.A. Fitzgerald, A.R. Kortan, S. Schuppler, M.A. Marcus, P.H. Citrin. Phys. Rev. Lett., 73, 3006 (1994).

[9] D.V. Yurasov, Yu.N. Drozdov, M.V. Shaleev, A.V. Novikov. Appl. Phys. Lett., 95, 151902 (2009).

[10] M.V. Shaleev, A.V. Novikov, D.V. Yurasov, J.M. Hartmann, O.A. Kuznetsov, D.N. Lobanov, Z.F. Krasilnik. Appl. Phys. Lett., 101, 151601 (2012).

[11] D. Pachinger, H. Groiss, H. Lichtenberger, J. Stang1, G. Hesser, F. Schäffler. Appl. Phys. Lett., 91, 233106 (2007).

[12] D. Pachinger, H. Lichtenberger, G. Chen, J. Stangl, G. Hesser, F. Schäffler. Thin Sol. Films, 517, 62 (2008).

[13] B.J. Spencer, P.W. Voorhees, J. Tersoff. Phys. Rev. B, 64, 253318 (2001).

[14] Ю.Н. Дроздов, Д.Н. Лобанов, А.И. Никифоров, А.В. Новиков, В.В. Ульянов, Д.В. Юрасов. Поверхность. РСНИ, № 7, 61 (2009).

[15] J.W. Matthews, A.E. Blakesley. J. Cryst. Growth, 27, 118 (1974).

Редактор Л.В. Шаронова

\section{Influence of surface roughness on the growth mode change of strained SiGe heterostructures from two-dimensional to three-dimensional}

\author{
A.V.Novikov ${ }^{+*}$, M.V. Shaleev ${ }^{+}$, D.V. Yurasov ${ }^{+*}$, \\ P.A. Yunin ${ }^{+}$ \\ + Institute for Physics of Microstructures, \\ Russian Academy of Sciences, \\ 603950 Nizhny Novgorod, Russia \\ * Lobachevsky State University of Nizhny Novgorod, \\ 603950 Nizhny Novgorod, Russia
}

\begin{abstract}
The influence of the surface microroughness on the critical thickness for two-dimensional growth of strained $\mathrm{SiGe}$ structures on $\mathrm{Si}(001)$ and $\mathrm{Ge}(001)$ substrates was investigated. The decrease of the two-dimentional (2D) growth critical thickness of $\mathrm{Ge}$ film in $\mathrm{Ge} / \mathrm{Si}$ lattices grown on $\mathrm{Si}(001)$ substrates was observed with increasing the number of lattice periods or decreasing the thickness of spacer Si layers. Such change in the 2D growth critical thickness is related to increase of the lattice surface roughness with the accumulation of elastic energy in compressed structures. Comparative studies of the growth of SiGe structures on $\mathrm{Si}(001)$ and $\mathrm{Ge}(001)$ substrates have shown that in wide range of SiGe layer compositions the critical thickness for 2D growth of tensile-strained layers is significantly larger than for compressed layers.
\end{abstract}

